\title{
An attempt to detect polarization effects in the envelope of $\gamma$ Cassiopeiae with the GI2T interferometer
}

\author{
K. Rousselet-Perraut ${ }^{1}$, F. Vakili ${ }^{2}$, D. Mourard ${ }^{2}$, F. Morand ${ }^{2}$, D. Bonneau ${ }^{2}$, and Ph. Stee ${ }^{2}$ \\ 1 IGRAP, Laboratoire d'Astronomie Spatiale, CNRS, Traverse du Siphon, BP. 8, 13376, Marseille Cedex 12, France \\ 2 Observatoire de la Côte d'Azur, Département Fresnel, CNRS URA 1361, 06460 Saint Vallier de Thiey, France
}

Received March 13; accepted September 16, 1996

\begin{abstract}
We report on the results of an observing run of $\gamma$ Cassiopeiae using the optical long baseline interferometer Grand Interféromètre à 2 Télescopes (GI2T) in the southern France equipped with film-sheet polarizers. Four interferometric baselines ranging from 20 to $40 \mathrm{~m}$ were obtained on this star during 1994 on September 5th and 6 th, with $\alpha$ Cephei as a calibrator. We aimed at detecting possible variations of the angular diameter of $\gamma$ Cas as a function of polarization directions parallel and orthogonal to the north-south baseline of the GI2T. We do not detect any variation of the visibility as a function of Hour Angle on $\alpha$ Cephei, neither on $\gamma$ Cas. We find an upper limit of 1.3 on the ratio of the angular diameters of the envelope of $\gamma$ Cas seen through polarizers parallel and perpendicular to the North-South direction of the GI2T's baseline. We discuss how this limit could constrain density laws in the wind of this Be star. We clearly resolve $\gamma$ Cas for increasing baselines in the red continuum whatever the polarization. Assuming the continuum flux originating from a photosphere surrounded by an elliptical gaussian envelope we estimate the extent of the latter as $(2.9 \pm 0.5)$ mas. This diameter agrees well with the $\mathrm{H} \alpha$ emitting envelope as obtained by other interferometers.
\end{abstract}

Key words: circumstellar matter - stars: Be - stars: individual $(\gamma$ Cas $)$ - stars: imaging — techniques: interferometric - polarization

\section{Introduction}

The merits of adding a polarimetric equipment to an optical interferometer has been recognized as early as 1970's from the observation of a few hot stars by the Narrabri intensity interferometer (Hanbury Brown 1974). Compared to polarimetric techniques, a polarimetric interferometer can be thought of as a slide-caliper which measures the distorsion of a scattering atmosphere seen through a linear polarizer (Cassinelli \& Hoffman 1975). In practice, the detection of this distorsion needs only two series of observations: the measure of the fringe visibility through a polarizer parallel to the interferometric baseline and perpendicular to it. As pointed by Cassinelli and Hoffman this possibility works even when the polarization of the atmosphere is axially symmetric -where classical polarimetry fails to detect any effect. This applies a fortiori to the intrinsec oblate circumstellar envelopes of Be stars, PMS objects or contact binaries (Poeckert \& Marlborough 1978; Fischer et al. 1996; Vakili 1981).

Polarization measurements are also relevant for calibrating systematic effects due to the differential rotation between incoming electromagnetic fields from separated telescopes (Beckers 1990; Tinbergen 1988). The present work aimed at comparing theoretical predictions of polarization effects in a stellar interferometer to actual data on the sky (Rousselet-Perraut et al. 1996). It was also initiated as part of an effort from our group to carry high spatial observations in polarized light with the GI2T new beamcombiner REGAIN (Mourard et al. 1994a). The bright Be star $\gamma$ Cas presents strong polarization properties a priori (Clarke 1990) and corresponds to a suitable target for evaluating the limits of such observations on the GI2T. In the following section we describe the instrumentation and data analysis which have been used for our study. The next section describes the interpretation of our interferometric measurements based on a simple model for the extended envelope of $\gamma$ Cas. The angular diameter of the envelope in the continuum is also compared to previous results of $\gamma$ Cas by the Mark III and the GI2T in the $\mathrm{H} \alpha$ emission line (Quirrenbach et al. 1993; Stee et al. 1995). In the last section our analysis is used to constrain the physics of the envelope of this star. Finally we conclude on a few scientific applications of the future polarimetric mode of GI2T + REGAIN.

Send offprint requests to: F. Vakili 


\section{Observations and data analysis}

\subsection{Instrumentation}

The status of the GI2T has been described in detail in two recent papers (Mourard et al. 1994b; Mourard et al. 1994c). The original beam-combiner of the GI2T was not equipped with polarizing optics (Bosc 1989). For the present study we have used the simplest form of a polarimeter consisting of two film-sheet linear polarizers. The directions of these polarizers were accurately set orthogonal in laboratory, and cross-talk effects calibrated as less than $3 \%$ in the continuum centered on $\lambda=660 \mathrm{~nm}$. During our observations we introduced the polarizers alternately, every 10 minutes on average, at the entrance of the GI2T's spectrograph (after beam recombination, Fig. 1, also see Fig. 5 of Mourard et al. 1994b). We sandwiched the recording of fringes in polarized light between series of data in natural light. This strategy aims at calibrating the effects of instrumental polarization on the visibility as a function of hour angle. It is worth emphasizing that in our polarimetric arrangement the two directions of polarization, noted $P_{\|}$and $P_{\perp}$, correspond to fixed orientations with respect to the coordinate system of the GI2T's central beam-combiner, i.e. the output pupil geometry. Actually the celestial field orientations of incoming beams from the north and south telescopes of the GI2T suffer a differential rotation which affects the fringe visibility as a function of the Hour Angle. The analytical expression of this degrading effect has been formulated by Mourard et al. (1994) and atttains at most $5 \%$ within the HA coverage of the present observations (Rousselet-Perraut et al. 1996). As discussed in Sect. 2.4, our observations are close enough to the meridian transit so as the visibilities estimated in $P_{\|}$and $P_{\perp}$ can be considered as measured parallel to the North-South direction.

\subsection{Observations}

We have observed $\gamma$ Cas (HD 5394, B0.5IVe, $m_{v}=2.7, \alpha$ $=0 \mathrm{~h} 51 \mathrm{mn}, \delta=60^{\circ} 11^{\prime}$ ) on September 5th and 6th 1994 with two baselines per night. The dispersed fringes, centered on $660 \mathrm{~nm}$, were recorded as series of data in the three states of polarizations, i.e. natural, parallel and perpendicular to the baseline. These data correspond to 20 ms short exposures from the CP40 photon-counting camera for freezing the atmospheric turbulence (Blazit 1987). All records were obtained at hour angles within -33 and $+96 \mathrm{mn}$ around the meridian transit in order to minimize the GI2T's instrumental losses of visibility (RousseletPerraut et al. 1996). At each baseline on $\gamma$ Cas, the records including the three polarizations were carried during 30 mn on average. We also observed the standard star $\alpha$ Cep (HD 203280, A7IV-V, $m_{v}=2.4, \alpha=21 \mathrm{~h} 51 \mathrm{mn}, \delta=$ $\left.62^{\circ} 10^{\prime}\right)$ for HA between -30 to $+80 \mathrm{mn}$ for calibrating our observations on $\gamma$ Cas (Tinbergen 1979).

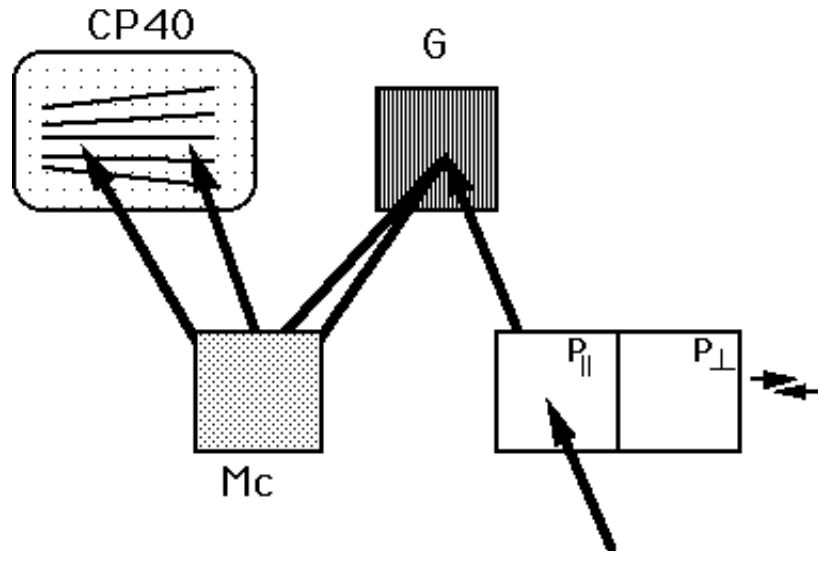

Fig. 1. Schematic layout of the optical set-up on the GI2T for observing $\gamma$ Cas in linearly polarized light. Sheet-film polarizers $P_{\|}$and $P_{\perp}$ (parallel and perpendicular to the baseline) are introduced alternately before the dispersing gratings $(\mathrm{G})$ of GI2T's spectrograph followed by the camera-mirror (Mc). The polarized interferograms are recorded on an intensified photon-counting detector (CP40)

Table 1. Journal of observations of $\gamma$ Cas and $\alpha$ Cep with the GI2T in September 1994. The columns give from left to right: the star, the date, the universal time (UT), the average Hour Angle (HA) in minutes respective to the meridian transit, the baseline projected on the sky in meters and the corresponding spatial frequency in cycles/arcsec for the continuum wavelength of $660 \mathrm{~nm}$.

\begin{tabular}{llllll}
\hline Star & Date & UT & $\langle$ HA $>$ & Baseline & Sp. Freq. \\
\hline$\gamma$ Cas & $94 / 9 / 6$ & $1: 12$ & -16 & 22.05 & 162.0 \\
$\prime \prime$ & $94 / 9 / 5$ & $1: 12$ & -20 & 27.75 & 203.8 \\
$\prime \prime$ & $94 / 9 / 6$ & $2: 12$ & +48 & 33.68 & 247.4 \\
$\prime \prime$ & $94 / 9 / 5$ & $2: 39$ & +65 & 39.60 & 290.9 \\
$\alpha$ Cep & $94 / 9 / 7$ & $22: 0$ & +20 & 27.50 & 202.0 \\
\hline
\end{tabular}

\subsection{Data processing}

We reduced the GI2T data following the method that we have developped for analysing multi-speckle interferograms of the GI2T in dispersed light (Mourard et al. 1994c). Since both $\gamma$ Cas and $\alpha$ Cep are bright objects, the cross-spectral density analysis of $20 \mathrm{~ms}$ short exposures was prefered to the usual spectral density for avoiding the so-called photon-centroiding-hole of the CP40 (Foy 1988; Blazit 1987).

In practice we cross-correlated fringes of two neighbouring narrow spectral channels taken in the continuum at $\lambda_{\mathrm{C}_{1}}=659.4 \mathrm{~nm}$ and $\lambda_{\mathrm{C}_{2}}=662.8 \mathrm{~nm}$. The spectral bandwidth per channel was $\Delta \lambda=3.42 \mathrm{~nm}$ in the range of the correlation limit for long baseline interferometry (Roddier 
1981). We computed the cross-spectral density by Fourier transforming the average cross-correlation as:

$C S_{\text {Cont }}(u)=<\left|\widetilde{I}_{\mathrm{C}_{1}}(u) \widetilde{I}_{\mathrm{C}_{2}}^{*}(u)\right|>$

where $<>$ refers to the ensemble average, ${ }^{*}$ to the complex conjugate and $u=B / \lambda$ to the spatial frequency, with $B$ the baseline and $\lambda$ the wavelength. Provided $\lambda_{\mathrm{C}_{1}} \sim \lambda_{\mathrm{C}_{2}}$, we can assume that $\widetilde{I}_{\mathrm{C}_{1}} \sim \widetilde{I}_{\mathrm{C}_{2}}$ (Petrov et al. 1986), thus:

$C S_{\text {Cont }}(u)=<\widetilde{I}_{\text {Cont }}^{2}(u)>=|\widetilde{O}(u)|^{2} T_{\text {Cont }}(u)$

where $|\widetilde{O}(u)|$ denotes the spatial power spectrum of the source and $T_{\text {Cont }}(u)$ the interferometric transfer function. We obtained the visibility estimates of our program stars by normalizing the high frequency energy to the atmospherically unbiased low frequency energy (Mourard et al. 1994c).

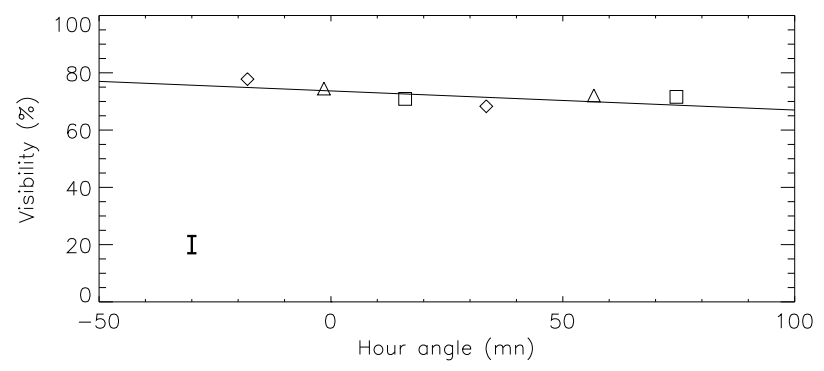

Fig. 2. Calibrated visibility points of $\alpha$ Cep for different states of polarization (natural light: squares, parallel to the baseline: diamonds, perpendicular to the baseline: triangles) as a function of hour angle. The typical error per visibility point $(1 \sigma \sim 5 \%)$ is given at the left-bottom

\subsection{Calibration of visibilities}

After reduction, we corrected raw visibilities at different baselines for the difference of transmission in the interferometric arms of the GI2T from actual fluxes recorded by the fine star-guider. We also corrected for the effect of differential field rotation between the afocal beams of the telescopes using the analytical expressions of the visibility loss versus the Hour Angle (Mourard et al. 1994a). This should enable us to detect an eventual variation of visibilities of $\gamma$ Cas during earth-rotation related to its intrinsec polarization. Figure 2 displays the corrected visibilities of $\alpha$ Cep versus HA where a slight trend was detected for increasing HA. This trend has also been noticed for other stars observed with the GI2T (Harmanec et al. 1996) and is believed to come from systematic instrumental effects of the interferometer. Using a linear model (Fig. 2) we correct for the decrease of visibilities on $\gamma$ Cas from those of $\alpha$ Cep. The residuals are on the order of $1-2 \%$, which is well below the atmospheric and photon noise of $5 \%$ obtained on the visibilities during this run. Therefore and in order to push further our analysis we decided to average, when possible, visibilities of a given polarization for a same baseline regardless of HA. Reduced error bars enabled us to check for partial resolution of $\gamma$ Cas in different polarizations for increasing baselines (Table 2).

Table 2. Calibrated visibilities in the continuum light of $\gamma$ Cas as a function of projected baseline on the sky (Col. 1) from GI2T observations. Each visibility is obtained by summing individual measurements at different hour angles. Visibilities are given in $\%( \pm 1 \sigma)$ for 3 different states of polarization: natural (Col. 2), linear parallel to the baseline (Col. 3) and linear perpendicular to it (Col. 4)

\begin{tabular}{llll}
\hline Baseline $(\mathrm{m})$ & $V_{\text {nat }}$ & $V_{\|}$ & $V_{\perp}$ \\
\hline 22.05 & $61.7 \pm 3.4$ & $60.5 \pm 4.2$ & $66.7 \pm 3.7$ \\
27.75 & $57.8 \pm 1.8$ & $62.7 \pm 2.2$ & $62.2 \pm 2.1$ \\
33.68 & $58.9 \pm 3.1$ & $54.9 \pm 3.1$ & $59.5 \pm 3.1$ \\
39.60 & $52.3 \pm 2.2$ & $55.8 \pm 2.3$ & $52.7 \pm 2.2$ \\
\hline
\end{tabular}

\section{Results on $\gamma$ Cas}

\subsection{The angular diameter of $\gamma$ Cas in the continuum}

The interpretation of the visibility points follows a simple model for $\gamma$ Cas where we consider the continuum source as a superposition of a central star and an extended circumstellar envelope. According to spectrophotometric estimates of $\gamma$ Cas, the underlying photosphere of this object has an angular diameter of .45 mas (Ochsenbein \& Halbwachs 1982) almost unresolved in the range of baselines achieved during this study. Formally, we estimate the equivalent uniform disk angular diameter of the envelope from the following model for the visibilities:

$V_{\text {cont }}(u)=\tau\left|F_{*} \frac{2 J_{1}\left(C \phi_{*} u\right)}{C \phi_{*} u}+F_{\mathrm{e}} \frac{2 J_{1}\left(C \phi_{\mathrm{e}} u\right)}{C \phi_{\mathrm{e}} u}\right|$.

$V_{\text {cont }}$ is the calibrated visibility at the spatial frequency $u$. $\tau$ is a normalization factor taking into account the attenuation of the zero baseline visibility by the GI2T (Mourard et al. 1994b). $J_{1}$ is the Bessel function of first degree. $\phi_{*}$ and $\phi_{\mathrm{e}}$ denote the equivalent uniform disk angular diameters of the star and the envelope in milliarcsecond and $C=1 / 65650000$ a conversion constant between $u$ and $\phi$. The fluxes $F_{*}$ and $F_{\mathrm{e}}$ of the star and the envelope are taken as .85 and .15 relative to the continuum emission at $660 \mathrm{~nm}$ according to Stee et al. (1995). This is obtained by considering the continuum photons of the extended envelope from free-free, free-bound emission and the scattering of the photospheric light in agreement with the models for $\gamma$ Cas (Poeckert \& Marlborough 1978; Stee et al. 1995). Note that we used the visibility model from Eq. (3) to interpret the visibilities for different polarizations. $V_{\text {nat }}$, 
$V_{\|}$and $V_{\perp}$ which correspond to the visibilities in natural, linearly polarized parallel and perpendicular to the baseline respectively. Figure 3 displays the visibility points in natural light and in linear polarizations as a function of the baseline according to Table 2 . The gap of visibilities at short baselines under 22.05 meter is due to the dimensions of the central hub of the GI2T which limits the minimum distance between the telescopes. This problem is overcome by setting the normalization factor $\tau$ as a free parameter of the model so as to obtain $V_{\text {cont }}(0) / \tau=1.0$. Finally we fit $\phi_{\mathrm{e}}$ in natural light by minimizing the $\chi^{2}$ function between model and observed visibilities from Eq. (3) and Table 2. We find $\phi_{\mathrm{e}}=4.10 \pm 0.50$ mas where the error on the angular diameter is obtained by differentiating $V_{\text {cont }}$ with respect to $\phi_{\mathrm{e}}$. This error takes into account the actual estimate of photon noise in computed power spectra (Eq. 2) and assumes that each $V_{\text {cont }}$ 's is an independant sample of the spatial power spectrum of $\gamma$ Cas measured by the GI2T.

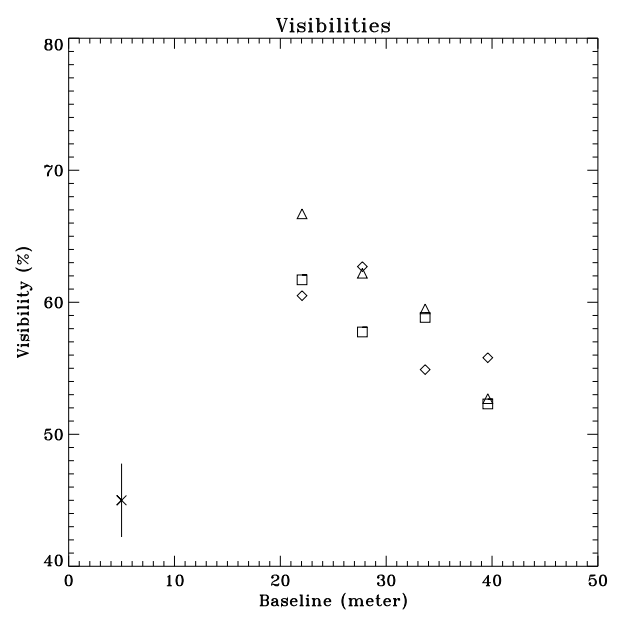

Fig. 3. Calibrated visibility points on $\gamma$ Cas as a function of baseline. The visibilities are given in $\%$ in the range of $40 \%$ to $80 \%$ for displaying an eventual variation of the visibilities related to polarization states (natural light: squares, linear parallel to the baseline: diamonds, polarized perpendicular to the baseline: triangles). The average error is given on the bottom-left of the plot

\subsection{The diameter of $\gamma$ Cas in linearly polarized light}

We applied the model of the previous section to the visibility points obtained in the two linear polarizations (Table 2). We found $\phi_{\|_{\mathrm{e}}}=4.09 \pm 0.50$ mas and $\phi_{\perp_{\mathrm{e}}}=4.13 \pm 0.50$ mas. By inspecting Fig. 3 one notes an apparent trend of $V_{\perp}$ visibilities (triangles) respective to $V_{\text {nat }}$ and $V_{\|}$which have a larger dispersion. We think that our limited accuracy on the visibilities (bottom-left of Fig. 3) can only set an upper limit to the ratio of these diameters by taking their maximum and minimum values at one $\sigma$, namely:

$\phi_{\perp_{\mathrm{e}}} / \phi_{\|_{\mathrm{e}}} \leq 1.3$.

Using 2D gaussians models to check a difference of flattening or major axes of elliptical envelopes in polarized light led us to the same conclusion. Note that in its present state -fixed north-south direction of resolution of the GI2T, our simple polarimetric experiment might miss detecting polarization effects in the envelope of gamma Cas if it happened to present special morphologies. Thus, for an unambiguous interpretation of such observations one would need other directions of resolution by adding a third telescope in the East-West direction or/and by using Earth-rotation synthesis. Also and ideally one could measure the whole set of Stokes visibilities in order to reconstruct or to model-fit intensity maps according to the classical definition of Stokes parameters (Shevgaonkar 1987).

\section{Discussion}

Bearing in mind the observational proof for the oblate envelope of $\gamma$ Cas we also examined elliptical gaussian models for interpreting our observed visibilities in natural light. The position angle of the major axis of this elliptical envelope was taken as $21^{\circ}$ to the East from the North according to Mark III observations (Quirrenbach et al. 1993). Our best fit leads to a major axis of $a_{\text {nat }}=2.9 \pm 0.5$ mas and a flattening of $e_{\text {nat }}=0.74 \pm 0.1$. Note that the gaussian model extent taken at $1 \sigma$ corresponds analytically to a smaller value than the uniform disk model diameter. The present major axes and flattening estimates are comparable to the results of the Mark III in $\mathrm{H} \alpha$, namely 3.2 mas and .72 for the major axis and the flatenning of $\gamma$ Cas envelope. On the other hand, from a previous interferometric run on the GI2T Stee et al. reported that the continuum emission of the envelope must essentially originate within 3-4 stellar radii (Stee et al. 1995), a result also inferred from IR studies (Waters \& Marlborough 1992). We find the continuum envelope twice as large as those estimates. The discrepancy might be explained by the fact that Stee et al. did not include the scattering of the photospheric light by the envelope for computing their continuum intensity map. This would mean that the free-free and free-bound emissions originate close to the central star whilst the scattering is chiefly produced in the outer regions of the envelope. In principle interferopolarimetric observations should have confirmed this conclusion if higher accuracies than our upper limit of 1.3 to $\phi_{\perp_{\mathrm{e}}} / \phi_{\|_{\mathrm{e}}}$ were obtained. One can reasonably speculate that improved interfero-polarimetry in the future should ultimately constrain the density and temperature distributions throughout the envelope among line-driven or rotationnally enhanced winds (Cassinelli \& Hoffman 1975; Poeckert \& Marlborough 1978). 


\section{Conclusion}

We have reported an attempt to observe polarization effects in the wind of $\gamma$ Cas. Though we have not attained our goal to detect such effects, we have resolved the circumstellar envelope of $\gamma$ Cas for the first time in the continuum. From this result we have estimated the extent of this envelope as $8-10$ times the diameter of the central star, comparable to the value found from interferometric observations in the $\mathrm{H} \alpha$ emission line (Thom et al. 1986; Quirrenbach et al. 1993; Stee et al. 1995).

From an instrumental point of view, we think that interfero-polarimetric accuracies could significantly be improved by recording simultaneously the fringe signal in different polarizations so as to minimize the dispersion of measured visibilities due to atmospheric turbulence and instrumental residuals. Such a differential scheme will be implemented as an observational mode of the future beamcombiner REGAIN of the GI2T to be operated by the end of 1997 (Rousselet-Perraut 1996). Interferometric polarimetry could therefore be extended to other classes of stars such as evolved red supergiants, magnetic stars, semi-contact binaries where the mutual reflection effect is at play. In fine, such observations could directly be interpreted in terms of physical parameters of the star being observed if adequate models built for interferometry become available. Such models should compute intensity maps and corresponding spatial power spectra in linear polarizations for a direct comparison with interferometric visibilities (Vakili et al. 1994).

Acknowledgements. We are grateful to Emeric Le Floch for his assistance during the observing runs. This work was partially supported by the PNHRAA french organisation from CNRSINSU.

\section{References}

Blazit A., 1987, PhD dissertation, Nice University

Bosc I., 1989, PhD dissertation, Nice University

Beckers J., 1990, Polarization effects in stellar interferometry, SPIE 1166, p. 165
Cassinelli J.P., Hoffman N.M., 1975, MNRAS 173, 789

Clarke D., 1990, A\&A 227, 151

Fischer O., Henning Th., Yorke H.W., 1996, A\&A 308, 863-885

Foy R., 1988, "The photon counting camera CP 40", in: Robinson L.B. (ed.) Instrumentation for GroundBased Optical Astronomy, Present and Future. New-York, Springer-Verlag, p. 589

Hanbury Brown R., Davis J., Allen L.R., 1974, MNRAS 168, 93

Mourard D., Tallon-Bosc I., Blazit A., et al., 1994a, REGAIN: A new optical beam-combiner for the GI2T, SPIE Vol. 2200, 593

Mourard D., Bosc I., Blazit A., et al., 1994b, A\&A 283, 705

Mourard D., Tallon-Bosc I., Rigal F., Vakili F., Bonneau D., Morand F., Stee Ph., 1994c, A\&A 288, 675

Ochsenbein F., Halbwachs J.L., A\&AS 47, 523

Petrov R., Roddier F., Aime C., 1986, J. Opt. Soc. Am. A3, 634

Poeckert R., Marlborough J.M., 1978, ApJ 220, 940

Quirrenbach A., Hummel C.A., Buscher D.F., et al., 1993, ApJ 416, L25

Roddier F., 1981, "Atmospheric limitations to HARI", Proceedings of ESO Conference, Garching, Germany

Rousselet-Perraut K., Vakili F., Mourard D., 1996, Opt. Eng. (in press)

Rousselet-Perraut K., 1996, PhD. Thesis, Université d'AixMarseille III

Shevgaonkar R.K., 1987, A\&A 176, 159-170

Stee P., De Araujo F.X., Vakili F., et al., 1995, A\&A 300, 219

Tinbergen J., 1979, A\&AS 35, 325

Tinbergen J., 1988, "Very Large Telescopes and their instrumentation", Proceedings of ESO Conference, Ulrich M.H. (ed.) 21-24 March, pp. 1267-1272

Thom C., Granès, Vakili F., 1986, A\&A 165, L13

Vakili F., 1981, A\&A 101, 352

Vakili F., Mourard D., Stee P., 1994, "Pulsation, Rotation and Mass-Loss in Early-type Stars", Proceedings of IAU Symposium 162. In: Balona L. et al. (eds.). Kluwer Acad. Publ.

Waters L.B.F.M., Marlborough J.M., 1992, A\&A 253, L25 